\title{
Joint Downlink Beamforming, Power Control, and Data Rate Allocation for DS-CDMA Mobile Radio with Multimedia Services
}

\author{
Ying-Chang Liang ${ }^{1}$, Francois P.S. Chin ${ }^{1}$ and K.J. Ray Lius \\ 1 Centre for Wireless Communications, 20 Science Park Road, \#02-34/37 TeleTech Park, \\ Singapore Science Park II, Singapore 117674 \\ Email: cwclyc@leonis.nus.edu.sg, cwccps@leonis.nus.edu.sg \\ ${ }^{2}$ Department of Electrical Engineering, University of Maryland, College Park, MD 20742 \\ Email: kjrliu@isr.umd.edu
}

\begin{abstract}
Power control and rate allocation are two fundamental issues for improving the spectrum efficiency of downlink transmission for wireless multimedia communications. Dounlink beamforming, on the other hand, can be used to suppress stronger interference induced by high rate users, thereby improving the system performance. In this paper, we present a joint downlink beamforming, power control and rate allocation technique suitable for DS-CDMA systems with multimedia services. To simplify the computational complexity, a practical rate allocation algorithm is also proposed. Computer simulation results are given to evaluate downlink capacity of DS-CDMA systems using base station antenna and the new algorithm proposed in this paper.
\end{abstract}

\section{Introduction}

The next generation mobile communication system will be required to provide wireless multimedia services, including voice, image, video, and high rate data. DSCDMA is one of the leading standards because of its advantages in system capacity and flexibility to support variable data rate services. Transmitting multimedia data over DS-CDMA channels generates stronger interference among users. Specifically, higher rate users are main sources of interference to the low rate users. This interference should be kept in certain low value in order to obtain reliable transmission quality. Spatial division multiple access (SDMA) [1], by which a plurality of antenna elements are equipped at the base station in order to receive and transmit data information from and to the desired user by using spatial diversities, is one of the effective techniques to suppress this kind of interference. The main operations in SDMA include uplink (from mobile station to base station) beamforming and downlink (from base station to mobile station) beamforming. Uplink beamforming is easily implemented as compared with its downlink counterpart. However, in practice, it is also desirable to increase downlink capac- ity in order to improve the whole system capacity. Also, downlink performance is even more important for the next generation communication systems in which wireless internet, video-on-demand and multimedia services are required [4].

For wireless multimedia communications, it is desired to design a system that dynamically adapts to the timevarying channel and traffic and, at the same time, delivers the different data streams according to their required quality-of-service (QOS). Power control and rate allocation $[3,4]$ are two fundamental techniques for providing reliable transmission over a time-varying channel.

When the base station is equipped with an antenna array, downlink beamforming weights should be designed using certain optimization criterion. In [2], for given downlink data rates, virtual uplink beamforming and power control technique (UBPCT) was proposed for generating downlink beamforming weights by minimizing total transmitted power while maintaining the SIR requirements. However, from the simulation examples shown in [2], given the system throughput, which is defined as the total downlink data rate, the total transmitted power is also dependent on the allocation of the downlink data rate. In this paper, we treat the data rate as another variable, and try to find out the optimal beamforming weights, transmitted powers and data rates by minimizing the total transmitted power while keeping the SIR at the target values. This is also equivalent to maximizing the Erlang capacity, which is defined as the number of active users [3].

The rest of this paper is organized as follows. In Section 2, downlink DS-CDMA transmission system is described. Section 3 addresses the problem of joint downlink beamforming, power control and data rate allocation, in which a practical rate allocation algorithm is proposed. Section 4 presents the computer simulations. Conclusions are drawn in Section 5. 


\section{System Description}

Single cell is considered in this paper. Suppose $N$ mobile users share the same base station in which a $M$ element antenna array is equipped. Narrowband signals are first spread to wideband signals using different spreading codes. A 2-layered code structure which consists of short spreading codes and long scrambling codes is adopt. The scrambling codes are assigned specifically to each cell in the downlink.

Multicode spreading scheme is used for multirate transmission. Suppose user $k$ is with normalized data rate $r_{d}(k)$, which is the ratio of $k$ th user's data rate to the basic data rate. Let $d_{c, k}^{(j)}(t)$ and $c_{c, k}^{(j)}(t)$ be the data signal and spreading signal of the $k$ th user's $j$ th code channel, respectively, and $P_{d, k}$ is the average transmitted signal power for one code channel of user $k$. The $k$ th signal to be transmitted to mobile user $k$ is given by

$$
s_{k}(t)=\sqrt{P_{d, k}} \sum_{j=1}^{r_{d}(k)} d_{c, k}^{(j)}(t) c_{c, k}^{(j)}(t)
$$

for $k=1, \cdots, N$, with

$$
\begin{gathered}
d_{c, k}^{(j)}(t)=\sum_{m=-\infty}^{\infty} d_{k}^{(j)}(m) u\left(\frac{t}{T}-m\right) \\
c_{c, k}^{(j)}(t)=\sum_{m=-\infty}^{\infty} \sum_{l=0}^{G-1} f(l+m G) c_{k}^{(j)}(l) u\left(\frac{t}{T_{c}}-l-m G\right)
\end{gathered}
$$

for $j=1, \cdots, r_{u}(k)$, where $f(m), m=\cdots,-1,0,1, \cdots$, represents the complex, scrambling sequences common to all users; $d_{k}^{(j)}(m), m=\cdots,-1,0,1, \cdots$, and $c_{k}(m), m=0, \cdots, G-1$, denote the data sequence and the $G$-chip, complex short spreading sequence of the $j$ th code channel of user $k$, respectively; $T$ and $T_{c}$ are the data symbol duration and chip duration, respectively; $u(t)=1$ for $0 \leq t<1$ and $u(t)=0$ elsewhere; and $G=T / T_{c}$ is the processing gain. We assume that $|f(l+m G)|=\left|c_{k}^{(j)}(l)\right|=1$, and $r_{d}(k)$ is a positive integer.

We consider per-user-per-weight (PUPW) beamforming scheme [2], and denote $\mathbf{w}_{d, j}$ as the downlink beamforming weight vector of user $j$. The received signal at mobile user $k$ is given by

$$
r_{k}(t)=\sum_{j=1}^{N} \sum_{i=1}^{L_{k}} \mathbf{w}_{d, j}^{H} \mathbf{h}_{d, k}^{(i)} s_{j}\left(t-\tau_{k}^{(i)}\right)+n_{k}(t)
$$

where $L_{k}$ is the number of resolvable paths of signals received at the $k$ th user, $\mathbf{h}_{d, k}^{(i)}$ and $\tau_{k}^{(i)}$ denote the downlink channel vector and time delay corresponding to the $i$ th path of user $k$, respectively; and $n_{k}(t)$ is additive white Gaussian noise (AWGN) received at mobile $k$. We assume the one-sided spectrum density of the AWGNs at mobiles are all equal to $N_{0}$.
For basic data rate users, the received signal is despread by using the spreading signal. For higher data rate users, several matched filters or correlators are employed in order to despread all code channels. The despread outputs are then passed to Rake combiner for signal detection. Given the downlink beamforming weight vectors, the signal-to-interference-plus-noise ratio, $S I R_{d, k}$, at the $k$ th user's combiner output can be found in [2]. In practice, SIR-based power control is also needed in order to compensate near-far problem. For same SIR requirement, we try to maintain $S I R_{d, k}=\gamma_{0}$ for each $k$, where $\gamma_{0}$ is the target SIR threshold, or

$$
\left(\mathbf{I}-\gamma_{0} \mathbf{F}_{d}\right) \mathbf{p}_{d}=\gamma_{0} \mathbf{g}_{d}
$$

where $\mathbf{F}_{d}=\mathbf{D F R}, \mathbf{p}_{d}=\left[\frac{P_{d, 1} T}{N_{0}}, \cdots, \frac{P_{d, N} T}{N_{0}}\right]^{T}$, and $\mathbf{g}_{d}=$

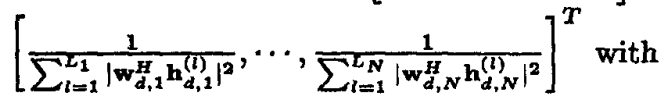

$\mathbf{D}=\operatorname{diag}\left[\frac{1}{\sum_{l=1}^{L_{1}}\left|\mathbf{w}_{d, 1}^{H} \mathbf{h}_{d, 1}^{(l)}\right|^{2}}, \cdots, \frac{1}{\sum_{l=1}^{L_{N}}\left|\mathbf{w}_{d, N}^{H} \mathbf{h}_{d, N}^{(l)}\right|^{2}}\right]$

$$
\mathbf{R}=\operatorname{diag}\left[r_{d}(1), \cdots, r_{d}(N)\right]
$$

and

$$
\begin{aligned}
& {[\mathbf{F}]_{i, j}=}
\end{aligned}
$$

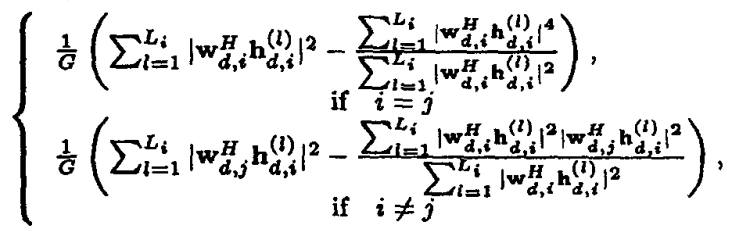

For a given set of channel responses and downlink beamforming weights, if and only if $\gamma_{0}<\frac{1}{\rho\left(F_{d}\right)}$, where $\rho\left(\mathbf{F}_{d}\right)$ is the spectral radius of $\mathbf{F}_{d}$, there exists a positive $\mathbf{p}_{d}=\gamma_{0}\left(\mathbf{I}-\gamma_{0} \mathbf{F}_{d}\right)^{-1} \mathbf{g}_{d}$ such that all SI $R_{d, k}$ 's are equal to $\gamma_{0}$.

For given downlink data rates, the objective of downlink beamforming is to choose a set of beamforming weights $\mathbf{w}_{d, k}$ 's, such that the total transmitted power, ${ }_{1}^{T} \mathbf{R}_{\mathbf{P}_{d}}$, is minimized while certain SIR requirement is satisfied.

Virtual uplink beamforming and power control technique (UBPCT) was proposed in [2] to derive the optimal beamforming weights. This technique is optimal in the sense that it not only takes into account the downlink data rate information, but also considers the orthogonality property of downlink codes.

\section{Joint downlink beamforming, power control and data rate al- location}

From (3), given downlink throughput, $\sum_{k=1}^{N} r_{d}(k)=N$, the total transmitted power is dependent on the data 
rate allocation. In this paper, maintaining the system throughput, we try to find out the optimal solution of the beamforming weights, transmitted powers and data rates such that the total transmitted power is minimized while certain SIR requirements are satisfied. A new virtual modified UBPCT is proposed to obtain the optimal solution.

\subsection{Virtual modified UBPCT}

The new technique consists of the following steps.

- (1.1) choose initial virtual uplink power and rate vectors;

- (1.2) compute the optimal weight vectors for given power and rate vectors;

- (1.3) compute and choose the rate and power vectors such that the total received power is minimal for given weight vectors;

- (1.4) iteratively update (1.2) and (1.3) until the power and weight vectors are converged. The converged rate and weight vectors are used as the downlink data rate and beamforming weight vectors.

The analysis of steps (1.2) and (1.3) with given data rate allocation is the same as that given in [2]. However, in step (1.3) of this new algorithm, it is required to obtain the optimal rate allocation such that the total virtual received power is minimal, which may involve complicated computations. In the next two subsections, we first analyze how the data rate allocation affects the required total transmitted power from the examples with small number of users, and find out some laws which guarantee the rate allocation. Then we propose a computational efficient algorithm for allocating the downlink data rates.

\subsection{2- and 3-user cases}

Fig.1 illustrates the required total transmitted powers and generated downlink beam patterns with optimal rate allocation for 2-user case. In Fig.1 (a), the two users are spatially well-separated, and the total transmitted power is minimal at $\left[r_{d}(1), r_{d}(2)\right]=[1.03,0.97]$. However, from Fig.1 (b), when the two users are spatially closed, the total transmitted power is slowly changing with respect to the rate allocation.

Further simulations were carried out for 3-user case. When the three users are spatially well-separated, the total transmitted power is minimal at around $\left[r_{d}(1), r_{d}(2), r_{d}(3)\right]=[1,1,1]$. However, if user 1 is spatially closed to user 3 , but far away from user 2 , the total transmitted power is minimal at around $r_{d}(2)=1.5$ and $r_{d}(1)+r_{d}(3)=1.5$, and it is also slowly changing with respect to the allocation for $r_{d}(1)$ and $r_{d}(3)$ so long as $r_{d}(1)+r_{d}(3)=1.5$. At the extreme, if all the three users are spatially closed, the total transmitted power is almost independent of the rate allocation.

\subsection{A rate allocation algorithm}

To remove the rate allocation ambiguity caused by the spatially closed users, we classify the spatially closed users as the same effective user, and denote $N_{e}$ as the number of effective users within the same sector. From the above examples, we may conclude the following.

- Given the downlink throughput, $N$, the data rate for each effective user is $N / N_{\mathrm{e}}$.

- The required total transmitted power is almost independent of the rate allocation among those users belonging to the same effective user, as long as the total rate of these user is equal to $N / N_{e}$.

It is required to make a decision which users belong to the same effective user. To this end, we propose to use the equivalent one-path channel vectors (EOCVs) as defined in [2]. Denote $\tilde{\mathbf{h}}_{d, k}$ as the EOCV of user $k$. We calculate $t_{i, k}=\frac{\left|\tilde{\mathbf{h}}_{d, i}^{H} \tilde{\mathbf{h}}_{d, k}\right|}{\left\|\mathbf{h}_{d, i}\right\|^{2}}$. Note $0<t_{i, k} \leq 1$, and the more close the two users are, the larger $t_{i, k}$ is. If $t_{i, k}$ is larger that certain value, say 0.8 , then users $i$ and $k$ belong to the same effective user; otherwise, they belong to two different effective users. The rate allocation algorithm involves the following steps.

- Calculate $t_{i, k}$ 's for $i, k=1, \cdots, N$, and detect the number of effective users, $N_{e}$;

- Uniformly allocate the data rate to each effective user;

- Uniformly allocate the data rate to each real user belonging to the same effective user.

With the above sub-optimal rate allocation, the virtual UBPCT of [2] can be used to generate the downlink beamforming weights directly. It is noted that the complexity of the new algorithm with rate allocation is comparable with that of the original virtual UBPCT. Also, once the rate allocation and downlink beamforming weights are available at the base station, fast transmit power control can be implemented according to the SIR values measured at the mobile terminal [2].

\section{Computer Simulations}

A 6-element ULA is equipped at the base station for each sector ( 3 sectors per cell). Consecutive antennas are spaced at half uplink wavelength. Single cell capacity is evaluated for both $\operatorname{TDD}\left(f_{u}=f_{d}=1.8 \mathrm{GHz}\right)$ and FDD $\left(f_{u}=1.8 \mathrm{GHz}, f_{d}=2.0 \mathrm{GHz}\right)$ duplex modes. Macrocell systems are considered in which the angular separation between each delay path of the same user is within $10^{\circ}$, and the angular spread for each delay path is $1^{\circ}$. Each user is with 2 delay paths and the processing gain is $G=16$. The SIR threshold is chosen to be $\gamma_{d, 0}=6.8 \mathrm{~dB}$, and the threshold for outage probability is chosen as $1 \%$. 
We compare three data rate allocations: (i) Uniform allocation: $r_{d}(k)=1$, for all $k$ 's; (ii) Random allocation: $r_{d}(k)$ 's are randomly chosen from $\{0.2,0.4, \cdots, 2.0\}$ with $\sum_{i=1}^{N} r_{d}(i)=N$; and (iii) Optimal allocation: $r_{d}(k)$ 's are allocated according to the algorithm proposed in Section 3.3. It is pointed out that $r_{d}(k)$ should be considered as "effective data rate" which takes into account the effect of activity parameter when $r_{d}(k)$ is a non-integer number [4].

Fig. 2 shows the outage probability with respect to different downlink throughput for TDD systems. It is seen that the virtual UBPCT with optimal rate allocations can support 35 users, while for random and uniform rate distributions, only 29 and 31 users can be supported.

Fig. 3 shows the results obtained for FDD systems. The number of users supported by the systems with random, uniform and optimal rate allocations are 27, 29 and 33 , respectively. The superiority of the UBPCT with optimal rate allocation is obvious, although there is certain capacity loss due to FDD.

\section{Conclusions}

A new rate allocation algorithm is proposed for downlink DS-CDMA mobile radio with multimedia services and base station antenna array. The complexity of the downlink beamforming with this rate allocation algorithm is comparable with the original virtual UBPCT without rate allocation, however, computer simulations have shown that, for both TDD and FDD modes, when the processing gain is 16 , the systems with optimal rate allocation can support 4 more users than the systems with uniform rate allocation, and 6 more users than those with random rate allocation.

\section{References}

[1] F. Rashid-Farrokhi, K.J.R. Liu and L. Tassiulas "Transmit beamforming and power control for cellular wireless systems," IEEE JSAC, vol.16, No.8, Oct. 1998, pp.1437-1449.

[2] Y.-C. Liang, F. Chin and K.J.R. Liu, "Downlink beamforming and power control for DS-CDMA mobile radio with multimedia services," Proc. of VTC'99-Fall, Amsterdam, The Netherland, Sep. 1999, pp.17-21.

[3] Y. Lu and R.W. Brodersen, "Integrating power control, error correction coding, and scheduling for CDMA downlink system," IEEE JSAC, 1999, vol.17, No.5, pp.978-989.

[4] D.G. Jeong and W.S. Jeon, "CDMA/TDD system for wireless multimedia services with traffic unbalance between uplink and downlink," IEEE JSAC, 1999, vol.17, No.5, pp.939-946.
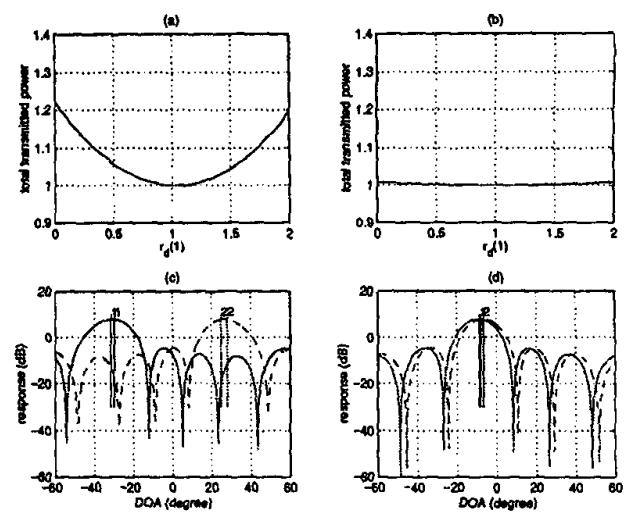

Figure 1: Comparison of total transmitted power and downlink beam patter with optimal rate allocation for 2-user case. (a) and (c): two users are spatially wellseparated; (b) and (d): two users are spatially closed.

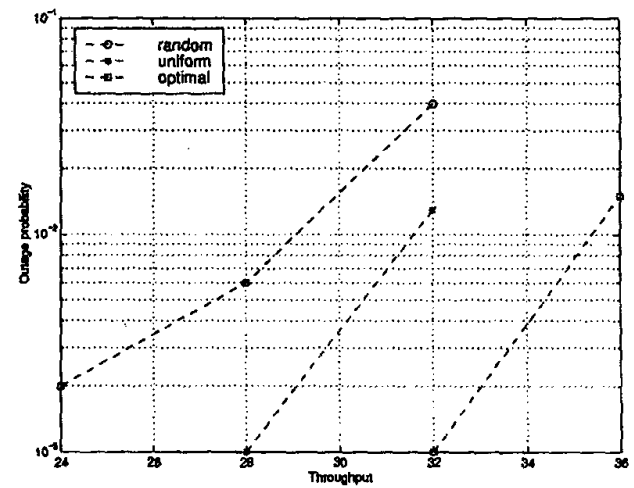

Figure 2: Outage probability of virtual UBPCT with different data rate allocation for TDD systems.

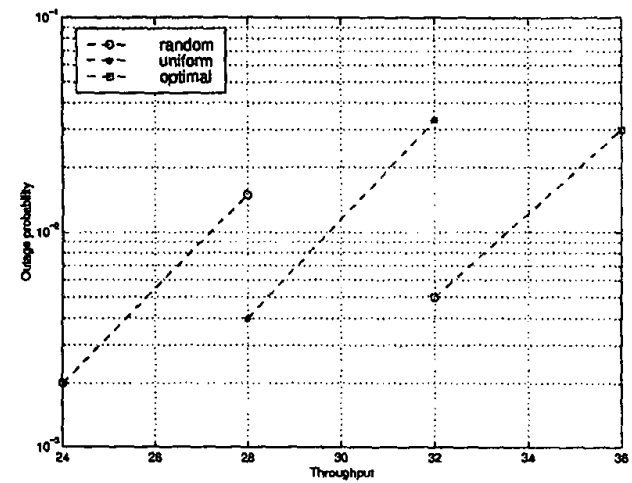

Figure 3: Outage probability of virtual UBPCT with different data rate allocation for FDD systems. 